\title{
A study of scheduling rules of flexible manufacturing systems: A simulation approach
}

\section{Sabuncuoglu}

To cite this article: I. Sabuncuoglu (1998) A study of scheduling rules of flexible manufacturing systems: A simulation approach, International Journal of Production Research, 36:2, 527-546, DOI: 10.1080/002075498193877

To link to this article: http://dx.doi.org/10.1080/002075498193877

$$
\text { 曲 Published online: } 14 \text { Nov } 2010 .
$$

Eubmit your article to this journal $₫$

Џlll Article views: 279

Q View related articles $\sqsubset$

Citing articles: 58 View citing articles 4 
INT. J. PROD. RES., 1998, vol. 36, NO. 2, 527-546

\title{
A study of scheduling rules of flexible manufacturing systems: a simulation approach
}

\section{SABUNCUOGLU†}

\begin{abstract}
This study examines the effects of scheduling rules on the performance of flexible manufacturing systems (FMSs). Several machine and AGV scheduling rules are tested against the mean flowtime criterion. In general, scheduling rules are widely used in practice ranging from direct applications as a stand-alone scheduling scheme to indirect application as a part of complicated scheduling systems. In this paper, we compare the rules under various experimental conditions by using an FMS simulation model. Our objective is to measure sensitivity of the rules to changes in processing time distributions, various levels of breakdown rates, and types of AGV priority schemes. A comprehensive bibliography is also presented in the paper.
\end{abstract}

\section{Introduction}

Flexible Manufacturing Systems (FMSs) have received increasing attention in the last decade. This is partly due to the fact that flexibility is required by manufacturing companies to stay in a highly competitive and changing business environment. Over the years, various types of FMSs have been designed and implemented worldwide. The existing implementations have demonstrated a number of benefits in terms of cost reductions and increased productivity, etc. However, these benefits are not easy to realize. Successful implementation of FMSs require solutions of various decision problems faced during design and operation stages of these systems.

It is generally known that an improvement in manufacturing productivity does not only depend on developments of high-tech physical components, but also the effective use of these advanced hardware systems. Specifically, scheduling and control algorithms are needed to run these expensive systems efficiently. However, the scheduling and control problem of an FMS is inherently complex and very difficult to solve, because an FMS is a highly dynamic system and its scheduling task requires additional resource considerations such as tools, fixtures, materials handling equipment, and limited buffer spaces. The flexibility inherent in these systems also further complicates the problems due to a large number of alternatives.

In recent years, there has been a growing interest in studying scheduling problems of FMSs. As a result numerous publications have emerged in the relevant literature. Basnet and Mize (1994) and Rachamadugu and Stecke (1994) provide the most upto-date and comprehensive review in this area. In general, there are basically three approaches in dealing with FMS scheduling problems: analytical techniques (Stecke 1983, Raman et al. 1989, Ulusoy and Bilge 1993) to simulation (Denzler and Boe 1987, Sabuncuoglu and Hommertzheim 1993) and artificial intelligence/expert systems (Kusiak and Chen 1988). We believe that research in each area is necessary for better understanding and solving the problems associated with the FMS. However,

$\dagger$ Bilkent University, Department of Industrial Engineering, Ankara, Turkey 06533. 
our focus in this paper will be on simulation-based experimental studies of the FMS scheduling problem. The problem is basically viewed as a dynamic scheduling problem and scheduling rules associated with a random FMS are analysed for the mean flowtime criterion by using a simulation model.

This paper is a sequel to Sabuncuoglu and Hommertzheim (1992a, b) and studies the scheduling rules under new experimental conditions. In Sabuncuoglu and Hommertzheim (1992a, b), we showed that flowtime performances of the rules are very sensitive to the variance of the operation time distribution. As compared to this study in which a normal distribution was used to control the variance of the processing time distribution, exponentially distributed processing times are used in this research. In general, the exponential distribution possesses a high degree of variability. In an FMS, completion of a transportation service by the AGV system creates job potentials for the machines. Similarly, completion of any machining operation generates an arrival to the AGV system. Since the duration of the operation time determines the inter-arrival time for the AGV system, the type and parameters of the processing time distribution may affect the relative performance of the AGV scheduling rules. Hence, one of our objectives in this paper is to observe differences, if any, in relative performances of the rules due to the changing operation time distribution. Moreover, we measure sensitivity of the results to stochastic events such as machine and AGV breakdowns. In this paper we also address the issue of order review release for FMSs. Specifically, we will demonstrate the importance of an AGV priority scheme for an effective input control for FMSs.

The rest of the paper is organized as follows. First, we give the relevant literature. This is followed in $\S 3$ by system considerations, and a description of the simulation model. In $\S 4$, we present the results of the simulation experiments. Finally, we conclude the paper with a summary of findings and suggestions for further research in $\S 5$.

\section{Relevant literature}

Scheduling rules are used to prioritize jobs on various resources (i.e. machines, material handling equipment, etc.) There is a wide base of literature available on these rules (Conway et al. 1967, Kiran and Smith 1984a, b, Panwalkar and Iskander 1977, Blackstone et al. 1982). The terms such as decision rules, priority rules and dispatching rules are often used interchangeably with scheduling rules. In any case, these rules are widely used in practice because of their simplicity and effectiveness in highly dynamic and stochastic manufacturing environments. They are not only used as stand alone scheduling mechanisms, but also employed as integral parts of analytical off-line scheduling algorithms (Sabuncuoglu and Karabuk 1995), knowledge-based scheduling systems (Wu and Wysk 1988) and iterative simulation based scheduling systems (Ishii and Talavage 1991).

Mackay et al. (1988) defined the real scheduling problem as 'how to schedule and dispatch work in such a way that many unstated and stated conflicting goals are satisfied using hard and soft information that is possibly incomplete, outdated, and erroneous'. The authors also reported that scheduling rules are especially used in dynamic situations where the systems experience frequent changes in the environment. But the general consensus is that no single rule is the best under all possible conditions. Also, effectiveness of a rule depends on a number of factors such as performance criteria, system load levels, due-date tightness, etc. For that reason, 
there are numerous studies in the literature which investigate the performances of rules under various operating conditions.

As compared to traditional job shop scheduling, there are relatively few experimental studies addressing scheduling rules in FMS environments. Moreover, the rules are mainly tested under machine dominated environments (Stecke and Solberg 1981, Denzler and Boe 1987, Choi and Malstrom 1988, Co et al. 1988, Ravi et al. 1991). That is, the main focus in these studies is on the machine scheduling rather than other elements of FMSs (i.e. a materials handling system).

One of the first simulation studies that tested the performance of scheduling rules for an AGV based material handling system is performed by Egbelu and Tanchoco (1984). In this study, several AGV scheduling rules are developed and their performances are measured via a simulation model. Later, Acree and Smith (1985) tested different cart selection and tool allocation rules. Their results found a significant different among the tool allocation rules. In another study, Montazeri and Van Wassenhove (1990) examined several machine scheduling rules and found that these rules have a large impact on the system performance. Tang et al. (1993) identify six decision rules for FMS scheduling involving operations among parts, machine, and AGVs. The Taguchi method is also used in the simulation experiments to study the relationship between the multiple performance measures and the rules.

Sabuncuoglu and Hommertzheim (1992a, b; 1993, 1995) study machine and AGV scheduling rules against various performance measures for a random type FMS. Their results indicate that some of the rules which perform well in job shop environments appear to carry over their superiority in FMSs as well. They also showed that the scheduling of AGVs is as important as the scheduling of machines. Moreover, the number of jobs released to the system (i.e. order review release mechanism) is found to be a significant factor affecting system performance. In fact, this study is an extension of the above works. In this paper, several machine and AGV scheduling rules are tested under a new set of experimental conditions.

As defined in Sabuncuoglu and Hommertzheim (1992a), two sets of rules (Table 1) are used in the experiments: (1) machine scheduling rules to prioritize the jobs on the machines and (2) AGV scheduling rules to dispatch AGVs. These rules are selected from the author's previous FMS studies. They are also frequently reported in the FMS literature. Among the rules, some of them use job based information (e.g. LWKR, FAFS) and operation-based information (i.e. SPT), whereas others utilize layout information (e.g. STD) and queue information (e.g. LQS, LOQS). Hence, they represent a wide range of rule possibilities.

\section{System considerations, simulation model and assumptions}

Figure 1 shows the layout of the hypothetical FMS studied in this paper. In the system, there are eight workstations with limited buffer capacities. There is also an input/output carousel where parts are mounted/unmounted to fixtures and palletized for transfer. The arriving parts are held in the carousel and allowed into the system on a FCFS basis as long as both an AGV and one queue space at the respective workcentre were available. In addition, there are two central buffer areas at which parts are temporarily stored to prevent system locking. Material handling is based on the AGV system. Other system details and the information about operating policies can be found in Sabuncuoglu and Hommertzheim (1992a). The FMS described above is a hypothetical system. The purpose of developing such a system is to use it as a 'test-bed' to measure performances of algorithms developed by the author for 
Symbol

Description

(1) Machine scheduling rules

SPT

SPT.TOT

SPT/TOT

LPT.TOT

LPT.TOT

LWKR

MWKR

FOPNR

MOPNR

FCFS

FAFS
Shortest processing time

Smallest value of operation time multiplied by total operation time

Smallest value of operation time divided by total operation time Largest value of operation time multiplied by total operation time Largest value of operation time divided by total operation time Least amount of work remaining

Most amount of work remaining

Fewest number of operations remaining

Most number of operations remaining

First come first served

First arrived first served

(2) AGV scheduling rules

\begin{tabular}{|c|c|}
\hline FCFS & First come first served \\
\hline LOQS & Largest output queue size \\
\hline LQS & Largest queue size \\
\hline STD & Shortest travel distance \\
\hline FOPNR & Fewest operations remaining \\
\hline LWKR & Least work remaining \\
\hline
\end{tabular}

Table 1. Machine and AGV scheduling rules.

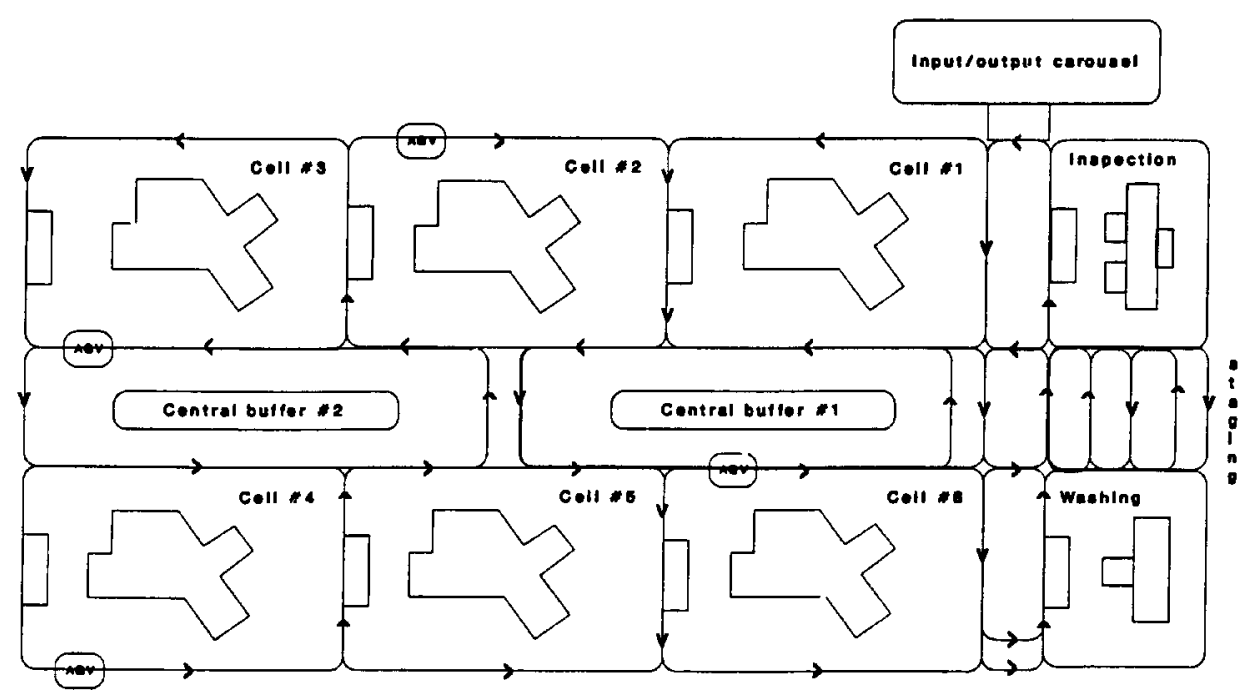

Figure 1. Schematic view of an FMS.

design and operation of FMSs. It was designed by considering several existing FMS configurations.

A discrete event simulation model of the system was written in SIMAN language (Pegden et al. 1990). The model was developed in such a way that three resources (machines, AGVs and limited buffer capacities) and their interactions were represented in detail. The job interarrival time and the processing times were exponentially distributed. The number of operations was determined by a discrete uniform 
distribution between one and six. The machine assignment was random and no job was allowed to visit the same machine more than once. All jobs visited the washing station. However, only $50 \%$ of the jobs were processed by the inspection station. In the simulation model, only two AGVs were employed. The reason for using a small number of AGVs is that the simulation execution time increased significantly when a large number of AGVs was operated.

A common random number variance reduction $\mathrm{CRN}$ technique was implemented to compare alternative rules or operating policies under identical conditions. In order to obtain consistent samples from the simulation model and eliminate the censored data problem, the jobs were numbered in the order of their arrival and the statistics were updated for these jobs only. The sample consists of 2000 jobs. Statistics for the first 300 jobs were discarded and the sample was collected for only the jobs numbered between 301 and 2300. The average simulation run length per replication was approximately 30000 minutes (or 3 months of continuous system operation).

\section{Analysis of simulation results}

The results of simulation experiments are analysed in three sections: exponentially distributed processing times, machine and AGV breakdowns, and job release methods.

\subsection{Exponentially distributed processing times}

In the exponential case, the scheduling rules were first compared under a set of standard conditions and then the sensitivity of results was measured by varying conditions (i.e. varying machine and AGV load levels, different queue capacities). Under the standard conditions, the buffer capacity is five and FCFS is used as the AGV rule to schedule two AGVs. As compared to the previous study (Sabuncuoglu and Hommertzheim 1992a) in which the normally distributed processing times were used, the average machine and AGV utilization rates were set to small values (approximately $75 \%$ and $82 \%$ ), because the system was saturated for most of the scheduling rules at high utilization rates due to the increased variance of exponential distribution.

\subsubsection{Standard experimental conditions}

First we had a close look at mean flowtime and its principal components (time in output queue, time in input queue, time in carousel, etc.) As seen in Table 2, a rule such as SPT which minimizes the mean flowtime also yields the best performances for its components. In the experiments, SPT.TOT produced the second best performance and this was followed by LWKR, SPT/TOT, FAFS, and FOPNR. Other rules such as LPT/TOT, LPT/TOT, MWKR, MOPNR, and MOPNR performed poorly. As can be noted, the system was even saturated for these rules. Among the AGV rules, STD yielded the minimum flowtime performances (Table 3). LQS performed competitively with STD by producing the second best performances. LWKR was ranked third and the other rules performed poorly. The relative performance of STD and LQS against the other measures is quite interesting. As can be seen in Table 3, STD minimized the average out-queue time (or waiting time of out-going parts), whereas LQS minimized the in-queue time (average waiting time of incoming parts). Knowing that an AGV handles only out-going parts, it was expected that the AGV rules would affect only the out-queue waiting time statistic. 


\begin{tabular}{lccccc}
\hline $\begin{array}{c}\text { Machine scheduling } \\
\text { rules }\end{array}$ & FM & MIQT & MOQT & MCT & MBT \\
\hline SPT & $308 \cdot 5$ & $122 \cdot 4$ & $39 \cdot 1$ & $44 \cdot 7$ & $0 \cdot 6$ \\
SPT/TOT & $373 \cdot 2$ & $147 \cdot 2$ & $50 \cdot 4$ & $70 \cdot 3$ & $1 \cdot 8$ \\
SPT.TOT & $318 \cdot 8$ & $128 \cdot 6$ & $41 \cdot 7$ & $45 \cdot 3$ & $1 \cdot 4$ \\
LPT/TOT & $*$ & $*$ & $*$ & $*$ & $*$ \\
LPT.TOT & $*$ & $*$ & $*$ & $*$ & $*$ \\
LWKR & $412 \cdot 9$ & $160 \cdot 4$ & $55 \cdot 3$ & $91 \cdot 7$ & $1 \cdot 9$ \\
MWKR & $*$ & $*$ & $*$ & $*$ & $*$ \\
FOPNR & $577 \cdot 5$ & $197 \cdot 8$ & $78 \cdot 9$ & $184 \cdot 7$ & $4 \cdot 9$ \\
MOPNR & $*$ & $*$ & $*$ & $*$ & $*$ \\
RANDOM & $*$ & $210 \cdot 9$ & $89 \cdot 2$ & $238 \cdot 6$ & $* 6$ \\
FCFS & $659 \cdot 5$ & $189 \cdot 7$ & $75 \cdot 8$ & $146 \cdot 2$ & $3 \cdot 8$ \\
FAFS & $524 \cdot 8$ & & & & $*$ \\
\hline
\end{tabular}

Nomenclature: MF mean flow time; MIQT, average queue time before operation; MOQT, average queue time after operation; MCT, average time on input/output carousel; MBT, average time being blocked.

* The system is saturated.

Table 2. Performances of machine scheduling rules when FCFS is used as the AGV scheduling rule.

\begin{tabular}{lcccrrr}
\hline \begin{tabular}{l} 
AGV scheduling \\
\multicolumn{1}{c}{ rules }
\end{tabular} & MF & MIQT & MOQT & NCT & MBT & TNB \\
\hline FCFS & $659 \cdot 5$ & $210 \cdot 5$ & $89 \cdot 1$ & $238 \cdot 6$ & $6 \cdot 5$ & 1300 \\
STD & $436 \cdot 7$ & $180 \cdot 8$ & $55 \cdot 7$ & $86 \cdot 7$ & $2 \cdot 3$ & 418 \\
LOQS & $684 \cdot 9$ & $218 \cdot 6$ & $85 \cdot 2$ & $251 \cdot 9$ & $7 \cdot 7$ & 1289 \\
LQS & $444 \cdot 3$ & $173 \cdot 0$ & $66 \cdot 4$ & $93 \cdot 7$ & $1 \cdot 5$ & 337 \\
LQKR & $491 \cdot 8$ & $175 \cdot 8$ & $67 \cdot 4$ & $137 \cdot 1$ & $2 \cdot 5$ & 488 \\
FOPNR & $592 \cdot 9$ & $189 \cdot 9$ & $77 \cdot 7$ & $200 \cdot 6$ & $4 \cdot 4$ & 768 \\
\hline
\end{tabular}

Nomenclature: TNB, total number of blockings; F, MIQT, MOQT, MCT, MBT are as defined in Table 2

Table 3. Performances of AGV scheduling rules when FCFS is used as the machine scheduling rule.

However, as it was observed from the performance of LQS, the average waiting time of in-queue time was also affected. This is again due to the fact that the same queue spaces are used by both incoming and out-going parts. Thus, the LQS rule which minimized the number of blocking, reduced the in-queue waiting time.

\subsubsection{Effect of the loading level on the relative performances of the scheduling rules}

In this section, the performance of the machine and AGV scheduling rule combinations are measured for varying system load levels. The results are also analysed to the ANOVA test for statistical significance.

Based on the previous results, the following four cases were identified (refer to Table 4 for the details): (1) both machines and AGV are highly loaded (case 1), 


\begin{tabular}{lcccc}
\hline \multicolumn{1}{c}{ Conditions } & Case 1 & Case 2 & Case 3 & Case 4 \\
\hline Machine utilization (\%) & $75 \cdot 0$ & $75 \cdot 0$ & 55 & 55 \\
AGV utilization (\%) & $82 \cdot 5$ & $61 \cdot 0$ & 81 & 55 \\
Inter-arrival time & $15 \cdot 00$ & $15 \cdot 00$ & $21 \cdot 00$ & $21 \cdot 00$ \\
Operation time & $19 \cdot 03$ & $19 \cdot 03$ & $19 \cdot 03$ & $19 \cdot 03$ \\
AGV speed (ft/min) & $65 \cdot 00$ & $86 \cdot 50$ & $42 \cdot 50$ & $61 \cdot 00$ \\
\hline
\end{tabular}

Table 4. Experimental conditions for testing machine and AGV scheduling rules under the varying load levels.

(2) only machines are highly loaded (case 2), (3) only AGVs are highly loaded (case 3 ), and (4) none of the subsystems are highly loaded (case 4). In each case considered, twelve rule combinations (four machine and three AGV scheduling rules) were tested under ten different experimental conditions (or replications). The machine and AGV load levels were set according to the procedure discussed in Sabuncuoglu and Hommertzheim (1992a). The scheduling rules were selected based on their performances in the previous sections. In each replication, the random numbers were fully synchronize to provide the same experimental conditions for each rule combination and obtain the desired variance reduction.

To analyse the relative performances of the rules, a randomized complete block design was adopted. According to this design, the machine and AGV rules were considered to be main factors. The treatments (or factor levels) were SPT, SPT/ TOT, SPT.TOT, and LWKR for the machine rules and STD, LQS, and LWKR for the AGV rules, respectively. Since the random numbers were fully synchronized for each rule, the different experimental conditions formed a blocking factor with 10 levels corresponding to each simulation replication.

In the ANOVA model, interactions between blocking and main factors were assumed to be negligible. This assumption was made due to the reason that the objective of the test was to identify the effects of main factors levels (i.e. machine and AGV rules) and interactions between them rather than finding interactions between replications (or random number seeds) and the scheduling rules. Also, the levels of the blocking factor were considered to be 'fixed' rather than random because the initial random number seed values were known in advance.

Since normality, equal variance, and randomness were three underlying assumptions for the above model, at each condition, validity of above assumptions were checked. Thus, depending on a violation of any one of the above assumptions, the original data set was transformed to another scale by using variance stabilization techniques. Finally, the analysis of variance procedure was followed by Duncan's multiple range test to rank the schedulng rules. All the statistical results reported in the paper were analysed using SYSTAT (Wilkinson 1987). The cases considered were as follows.

Case 1. Machines and AGVs are highly loaded. Since machines and AGVs were quite loaded, it was expected that both machine and AGV rules would have significant effects on the system performance. The ANOVA table displayed in Table 5 confirmed our expectations. As also illustrated in Figs 2 and 3, the blocking factor was also significant. This indicated that blocking was helpful in improving the precision during the comparison of rules. Finally we noted that there was no interaction between machine and AGV rules (Fig. 4). 


\begin{tabular}{|c|c|c|c|c|}
\hline Source of variation & $\begin{array}{l}\text { Sum of } \\
\text { squares }\end{array}$ & $\begin{array}{c}\text { Degrees of freedom } \\
(\mathrm{df})\end{array}$ & $\begin{array}{l}\text { Mean } \\
\text { square }\end{array}$ & F-ratio \\
\hline \multicolumn{5}{|c|}{ Case 1} \\
\hline $\mathrm{MCH}$ & $92021 \cdot 57$ & 3 & $30673 \cdot 86$ & $324 \cdot 20$ * \\
\hline AGV & $18299 \cdot 80$ & 2 & $9149 \cdot 90$ & $6.71 *$ \\
\hline $\mathrm{MCH}^{*} A G V$ & 288.06 & 6 & $48 \cdot 01$ & $0 \cdot 80$ \\
\hline BLOCK & $61008 \cdot 60$ & 9 & $6778 \cdot 73$ & $71 \cdot 65^{*}$ \\
\hline ERROR & $9366 \cdot 80$ & 99 & $94 \cdot 61$ & \\
\hline \multicolumn{5}{|c|}{ Case 2} \\
\hline $\mathrm{MCH}$ & $67289 \cdot 01$ & 3 & $22429 \cdot 67$ & $59 \cdot 56^{*}$ \\
\hline $\mathrm{AGV}$ & $207 \cdot 54$ & 2 & $103 \cdot 77$ & $2 \cdot 26$ \\
\hline $\mathrm{MCH}^{*} A G V$ & $24642 \cdot 48$ & 2 & $9149 \cdot 90$ & $96 \cdot 71 *$ \\
\hline BLOCK & $34558 \cdot 70$ & 9 & $3839 \cdot 73$ & $71 \cdot 65^{*}$ \\
\hline ERROR & $4541 \cdot 05$ & 99 & $45 \cdot 86$ & \\
\hline \multicolumn{5}{|c|}{ Case 3} \\
\hline $\mathrm{MCH}$ & $2928 \cdot 09$ & 3 & $979 \cdot 36$ & $10 \cdot 35^{*}$ \\
\hline AGV & $12098 \cdot 87$ & 2 & $6049 \cdot 44$ & $96 \cdot 71 *$ \\
\hline $\mathrm{MCH}^{*} A G V$ & $70 \cdot 80$ & 6 & $11 \cdot 80$ & $0 \cdot 72$ \\
\hline BLOCK & $10688 \cdot 27$ & 9 & $1187 \cdot 60$ & $72 \cdot 73 *$ \\
\hline ERROR & $1627 \cdot 80$ & 99 & $16 \cdot 44$ & \\
\hline \multicolumn{5}{|c|}{ Case 4} \\
\hline $\mathrm{MCH}$ & $2789 \cdot 86$ & 3 & $929 \cdot 95$ & $444.95 *$ \\
\hline $\mathrm{AGV}$ & $69 \cdot 85$ & 2 & $34 \cdot 92$ & $16 \cdot 70^{*}$ \\
\hline $\mathrm{MCH}^{*} A G V$ & $19 \cdot 13$ & 6 & $3 \cdot 29$ & $1 \cdot 52$ \\
\hline BLOCK & $3607 \cdot 60$ & 9 & $400 \cdot 84$ & $191 \cdot 78 *$ \\
\hline ERROR & $206 \cdot 91$ & 99 & $2 \cdot 90$ & \\
\hline
\end{tabular}

* indicates that test statistic is significant at 5\% level

Table 5. ANOVA table (using original data set).

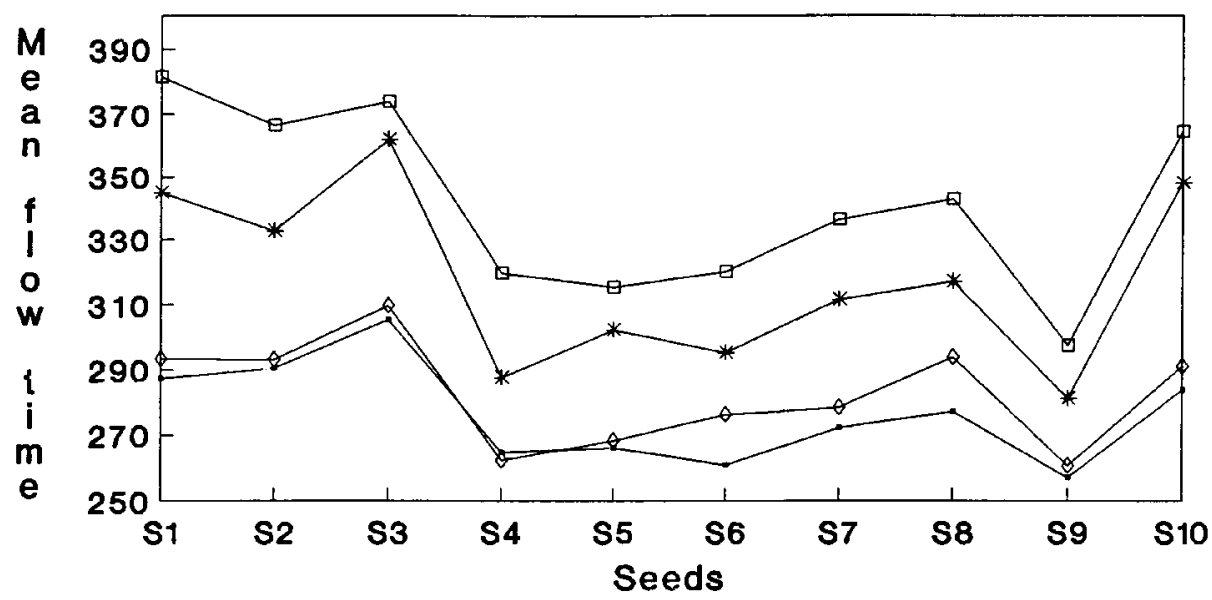

—SP1 $\rightarrow$ SPT/TOT $-\theta$ SP1.TOT $\because$ LWKR

Figure 2. Performances of machine rules at varying experimental conditions defined by seeds. 


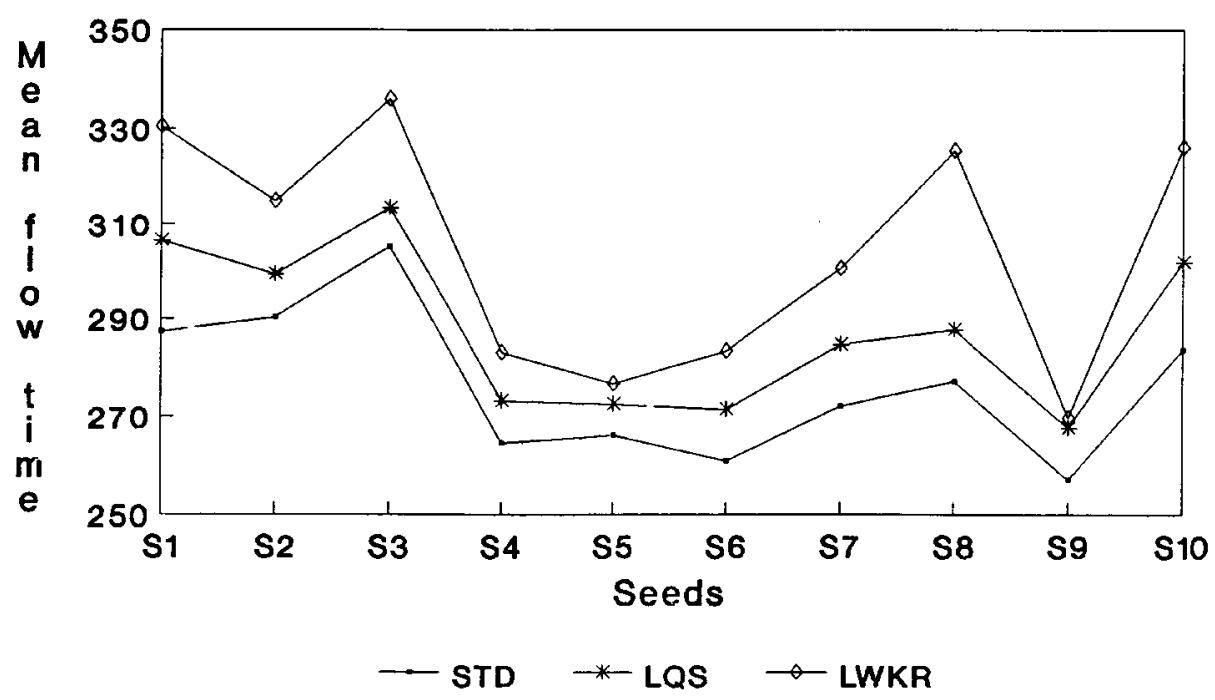

Figure 3. Performances of AGV rules at varying experimental conditions defined by seeds.

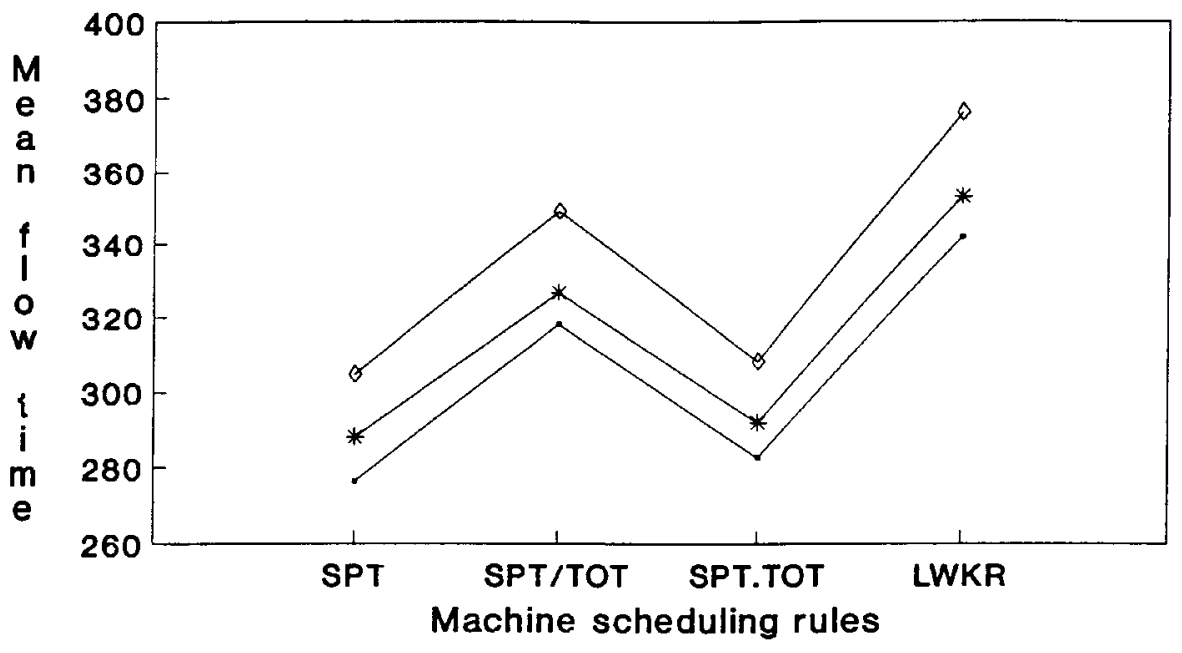

STD $\quad \rightarrow$ LQS $\rightarrow$ LWKR

Figure 4. Mean flow time performances of the rules.

During the analysis for checking model adequacy, it was noticed that the common variance assumption was violated for the machine scheduling rules. In general, the F-test is robust against unequal variances when the sample size is approximately equal. However, this unequal variance can be stabilized by using some suitable transformation. In our experiments, the standard deviation was proportional to the mean. Hence, the logarithmic transformation was applied to the original data set. The results did not change after transformations (i.e. machine, $\mathrm{AGV}$, and blocking were still significant at the 5\% level). 


\begin{tabular}{lcccc}
\hline & Case 1 & Case 2 & Case 3 & Case 4 \\
\hline MCH rule & SPT and & SPT and & SPT and & SPT and \\
AGV rule & SPT.TOT & SPT.TOT & SPT.TOT & SPT.TOT \\
& STD & STD & STD & STD \\
\hline
\end{tabular}

Table 6. Results of the Duncan's tests.

Finally, Duncan's multiple range test was applied to the modified data set (Table 6). The results indicated that performances of SPT and SPT.TOT were not statistically different. However, these two machine scheduling rules were better than SPT/TOT and LWKR. Among the AGV rules, STD was better than LQS which was followed by LWKR.

Case 2. Only machines are highly loaded. Since the machines were relatively more loaded than AGVs, it was expected that only machining system was significant. The ANOVA test confirmed the above expectation. Duncan's multiple range test results indicated that SPT and SPT.TOT were still the best two rules. SPT was slightly better than SPT.TOT. STD was the best AGV rule.

Case 3. Only AGVs are highly loaded. Since the AGVs were relatively more loaded than the machines, it was expected that only the AGV rules were significant. But the test results indicated that the machine rules were also significant. According to Duncan's test, STD was better than LQS which was followed by LWKR. Among the machine rules tested, both SPT and SPT.TOT performed better than LWKR. But differences between these two rules were not statistically significant.

Case 4. Machine and AGVs are lightly loaded. Since machines and AGVs were lightly loaded, it was expected that none of the machine nor AGV rules were significantly different. However, the ANOVA results (Table 5) indicated that both the machine and the AGV rules were significant. This interesting finding can be explained by the presence of significant blocking effect. Because blocking (by CRN) must be so effective that it reduced noise in the error term and hence magnified differences between the rules.

To justify the above conclusion, simulation experiments were repeated using a completely randomized design (i.e. a set of ten independent simulation runs was taken for each rule combination). Under this design policy, the ANOVA results showed no evidence for a significant difference between AGV rules. This clearly indicates that small differences in the performances of rules can even be detected by the randomized block design. The results of Duncan's test indicated that STD was better than LQS and LWKR. Among the machine rules, SPT was the best rule and this was followed by SPT.TOT, SPT/TOT, and LWKR.

\subsubsection{The effect of queue capacity on the relative performances of the scheduling rules}

We also examine the effect of different queue capacities on the relative performances of scheduling rules. The mean flowtime performances of three machine and AGV scheduling rules combinations at varying queue capacities are shown in 


\begin{tabular}{llccc}
\hline & & \multicolumn{3}{c}{ Machine rules } \\
\cline { 3 - 5 } & \multicolumn{1}{c}{ AGV rules } & SPT & SPT.TOT & LWKR \\
\hline Q=6 & STD & $269 \cdot 9$ & $272 \cdot 9$ & $337 \cdot 2$ \\
& LQS & $287 \cdot 5$ & $286 \cdot 2$ & $338 \cdot 6$ \\
& LWKR & $292 \cdot 9$ & $300 \cdot 0$ & $248 \cdot 6$ \\
Q=5 & STD & $279 \cdot 5$ & $282 \cdot 9$ & $300 \cdot 1$ \\
& LQS & $293 \cdot 5$ & $300 \cdot 1$ & $366 \cdot 8$ \\
QWE & LWKR & $313 \cdot 4$ & $315 \cdot 4$ & $380 \cdot 4$ \\
& STD & $332 \cdot 2$ & $341 \cdot 0$ & $411 \cdot 8$ \\
& LQS & $329 \cdot 9$ & $344 \cdot 9$ & $404 \cdot 9$ \\
Q $=3$ & LWKR & $383 \cdot 6$ & $395 \cdot 0$ & $500 \cdot 5$ \\
& STD & $*$ & $*$ & $*$ \\
& LQS & $579 \cdot 2$ & $581 \cdot 3$ & $*$ \\
\hline
\end{tabular}

* The system was saturated at $\mathrm{Q}=2$ for all rule combinations

Table 7. Mean flowtime performance of machine and AGV scheduling rules at varying queue capacities.

Table 7. Recall that under the standard experimental conditions, the queue capacity at each workcentre was five.

Among the machine scheduling rules, SPT and SPT.TOT provided a substantial flowtime improvement over the other machine scheduling rules when the queue capacity was reduced. SPT appeared to be better than the SPT.TOT rule. On the other side, among the AGV rules tested, STD and LQS minimized the mean flowtime. However, their relative performances changed as the queue capacity was varied. From Table 7, it is clear that LQS was a very effective AGV rule when the queue capacity was reduced. In other words, STD became better when the queue capacity increased. Again, the system saturated for most of the rule combinations when the queue size was three. Only the machine rules SPT and SPT.TOT with the LQS rule combination were survived at that queue capacity level.

\subsection{An analysis of scheduling rules under machine and AGV breakdown}

In the previous sections, machines and AGVs were assumed to be available continuously. However, manufacturing systems are subject to various interruptions such as machine failures, AGV breakdowns, tool failure, etc. In this section, scheduling rules are tested under the possibility of machine and AGV breakdowns. To model the breakdowns, it is necessary to specify the time between failure, resource type (i.e. machine and AGV number), and the extent of interruption (i.e. repair time). In the simulation model, values of inter-failure times were sampled from the exponential distribution. Repair times were constant and their values were 30 and 10 min for machines and AGVs, respectively. The resource type was determined using the discrete uniform distribution so that each individual resource of a given type (machine or AGV) experienced similar failure and down time. Again, in order to provide the same experimental conditions for each rule, a separate random seed was allocated for each parameter. Current values of the parameters can be found in 


\begin{tabular}{ccr}
\hline Down time $(\%)$ & Machine & AGV \\
\hline 1 & $371 \cdot 25$ & 495.00 \\
3 & $121 \cdot 25$ & 161.67 \\
5 & $71 \cdot 25$ & 95.00 \\
7 & $49 \cdot 82$ & 66.43 \\
10 & 33.75 & 45.00 \\
\hline
\end{tabular}

Table 8. Inter-failure time parameters for machines and AGVs (min).

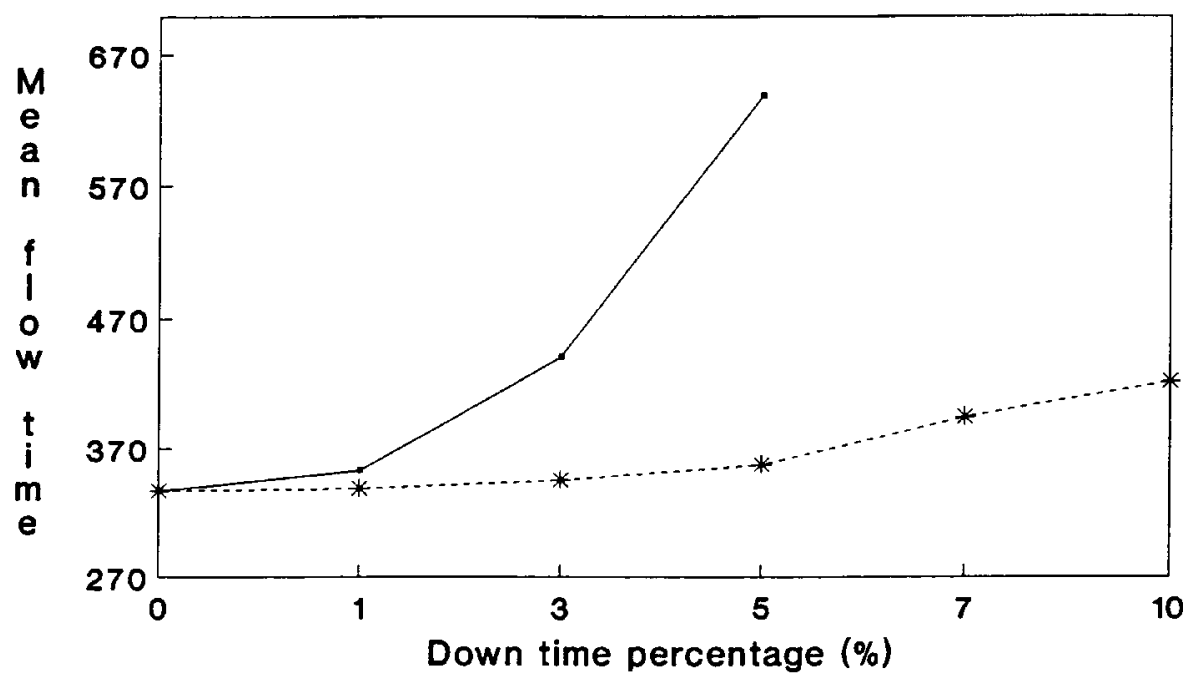

Figure 5. Effects of machine and AGV breakdowns- on the system performance.

Table 8. Four AGV and five machine rules were considered in the simulation experiments.

Each rule combination was tested under the varying level of down time percentage. The level of down time was controlled by changing the inter-failure distributions. In order to distinguish the impact of machine and AGV breakdown on the flowtime performance of the rules, machine and AGV breakdown cases were considered separately. The results of the simulation experiments are summarized in Tables 9 and 10 .

In general, the performance of the rules deteriorated as the down time percentage increased. As can be noted in Fig. 5, the adverse effect of machine failure on the system performance was more than the AGV failure. Under the machine breakdown (Table 9), SPT was the best machine scheduling rule. The SPT.TOT also performed well and this was followed by LWKR and SPT $\backslash$ TOT. The FCFS rule displayed the poorest performance. Among the AGV rules tested, LQS performed better than other rules. This may be due to the fact that LQS responds faster than STD and 1 as a result of increasing downtime percentage. We also noted that the system was saturated (or became unstable) for most of the rule combinations when the down time percentage was $>3 \%$. 


\begin{tabular}{|c|c|c|c|c|c|}
\hline \multirow[b]{2}{*}{ Down time } & \multirow[b]{2}{*}{ Machine rules } & \multicolumn{4}{|c|}{ AGV rules } \\
\hline & & FCFS & STD & LQS & LWKR \\
\hline \multirow[t]{5}{*}{$0 \%$} & SPT & $345 \cdot 0$ & $338 \cdot 1$ & $336 \cdot 8$ & $354 \cdot 9$ \\
\hline & SPT/TOT & $397 \cdot 7$ & $380 \cdot 8$ & $364 \cdot 1$ & $412 \cdot 3$ \\
\hline & SPT.TOT & $355 \cdot 2$ & $345 \cdot 0$ & $344 \cdot 0$ & $358 \cdot 1$ \\
\hline & LWKR & $408 \cdot 3$ & $374 \cdot 5$ & $361 \cdot 4$ & $387 \cdot 3$ \\
\hline & FCFS & $477 \cdot 7$ & 421.9 & $384 \cdot 9$ & $407 \cdot 0$ \\
\hline \multirow[t]{5}{*}{$1 \%$} & SPT & $394 \cdot 7$ & $370 \cdot 7$ & $352 \cdot 6$ & $386 \cdot 0$ \\
\hline & SPT/TOT & $459 \cdot 5$ & $417 \cdot 7$ & $394 \cdot 7$ & $474 \cdot 3$ \\
\hline & SPT.TOT & $407 \cdot 9$ & $377 \cdot 4$ & $368 \cdot 7$ & $394 \cdot 6$ \\
\hline & LWKR & $436 \cdot 0$ & $398 \cdot 6$ & $383 \cdot 7$ & $440 \cdot 7$ \\
\hline & FCFS & $643 \cdot 1$ & $447 \cdot 1$ & $440 \cdot 7$ & $564 \cdot 2$ \\
\hline \multirow[t]{5}{*}{$3 \%$} & SPT & $*$ & $*$ & $441 \cdot 4$ & $552 \cdot 8$ \\
\hline & SPT/TOT & $*$ & $*$ & $498 \cdot 6$ & $*$ \\
\hline & SPT.TOT & $*$ & $*$ & $469 \cdot 2$ & $532 \cdot 6$ \\
\hline & LWKR & $*$ & $*$ & $501 \cdot 3$ & $*$ \\
\hline & FCFS & $*$ & $584 \cdot 3$ & $524 \cdot 5$ & $*$ \\
\hline \multirow[t]{5}{*}{$5 \%$} & SPT & $*$ & * & $638 \cdot 2$ & $*$ \\
\hline & SPT/TOT & $*$ & $*$ & $*$ & $*$ \\
\hline & SPT.TOT & $*$ & $*$ & * & $*$ \\
\hline & LWKR & $*$ & $*$ & $*$ & $*$ \\
\hline & FCFS & $*$ & $*$ & * & $*$ \\
\hline \multirow[t]{5}{*}{$7 \%$} & SPT & $*$ & $*$ & * & * \\
\hline & SPT/TOT & $*$ & $*$ & $*$ & $*$ \\
\hline & SPT.TOT & $*$ & $*$ & $*$ & $*$ \\
\hline & LWKR & $*$ & $*$ & $*$ & $*$ \\
\hline & FCFS & $*$ & $*$ & $*$ & $*$ \\
\hline \multirow{5}{*}{$10 \%$} & SPT & $*$ & $*$ & $*$ & $*$ \\
\hline & SPT/TOT & $*$ & $*$ & $*$ & $*$ \\
\hline & SPT.TOT & $*$ & $*$ & $*$ & $*$ \\
\hline & LWKR & $*$ & $*$ & $*$ & $*$ \\
\hline & FCFS & $*$ & $*$ & $*$ & $*$ \\
\hline
\end{tabular}

* The system was saturated.

Table 9. Performance of the rules at varying machine breakdown rates.

Under the AGV breakdown (Table 10), SPT was still the best machine scheduling rule. However, SPT.TOT_produced better results than SPT with the combination of FCFS and LWKR when downtime percentage was high. In contrast to machine breakdown, SPT \TOT resulted in better performances than LWKR. Again, the FCFS was the worst of all. On the other side, LQS and SDT were better AGV rules than FCFS and LWKR. In general, LQS produced better results than STD. However, STD minimized the mean flowtime with the combination of SPT and SPT \TOT when AGV downtime was increased (7\% or above). In conclusion, the results obtained in this section are consistent with the previous conclusions.

\subsection{Effect of changing the AGV priority scheme on flowtime}

In the system studied, a part waits for an AGV either in the workcentre queue, in the central buffer, or in the input/output carousel. In the simulation runs reported 


\begin{tabular}{|c|c|c|c|c|c|}
\hline \multirow[b]{2}{*}{ Down time } & \multirow[b]{2}{*}{ Machine rules } & \multicolumn{4}{|c|}{ AGV rules } \\
\hline & & FCFS & STD & LQS & LWKR \\
\hline \multirow[t]{5}{*}{$0 \%$} & SPT & $345 \cdot 0$ & $338 \cdot 1$ & $336 \cdot 8$ & $354 \cdot 9$ \\
\hline & SPT/TOT & $397 \cdot 7$ & $380 \cdot 8$ & $364 \cdot 1$ & $412 \cdot 3$ \\
\hline & SPT.TOT & $355 \cdot 2$ & $345 \cdot 0$ & $344 \cdot 0$ & $358 \cdot 1$ \\
\hline & LWKR & $408 \cdot 3$ & $374 \cdot 5$ & $361 \cdot 4$ & $387 \cdot 3$ \\
\hline & FCFS & $477 \cdot 7$ & $421 \cdot 9$ & $384 \cdot 9$ & $407 \cdot 0$ \\
\hline \multirow[t]{5}{*}{$1 \%$} & SPT & $365 \cdot 6$ & $338 \cdot 6$ & $338 \cdot 1$ & $368 \cdot 6$ \\
\hline & SPT/TOT & $412 \cdot 8$ & $373 \cdot 6$ & $362 \cdot 6$ & $433 \cdot 5$ \\
\hline & SPT.TOT & $381 \cdot 4$ & $377 \cdot 4$ & $368 \cdot 7$ & $394 \cdot 6$ \\
\hline & LWKR & $461 \cdot 8$ & $372 \cdot 5$ & $368 \cdot 6$ & $388 \cdot 9$ \\
\hline & FCFS & $461 \cdot 8$ & $430 \cdot 8$ & $400 \cdot 8$ & $529 \cdot 5$ \\
\hline \multirow[t]{5}{*}{$3 \%$} & SPT & $372 \cdot 0$ & $351 \cdot 5$ & $345 \cdot 1$ & $392 \cdot 9$ \\
\hline & SPT/TOT & $463 \cdot 8$ & $412 \cdot 0$ & $376 \cdot 9$ & $505 \cdot 8$ \\
\hline & SPT.TOT & $386 \cdot 2$ & $381 \cdot 3$ & $362 \cdot 7$ & $389 \cdot 0$ \\
\hline & LWKR & $545 \cdot 7$ & $384 \cdot 3$ & $377 \cdot 2$ & $416 \cdot 8$ \\
\hline & FCFS & $545 \cdot 8$ & $443 \cdot 9$ & $407 \cdot 4$ & $543 \cdot 3$ \\
\hline \multirow[t]{5}{*}{$5 \%$} & SPT & $393 \cdot 0$ & $355 \cdot 4$ & $357 \cdot 1$ & $424 \cdot 6$ \\
\hline & SPT/TOT & $498 \cdot 9$ & $423 \cdot 9$ & $382 \cdot 9$ & $*$ \\
\hline & SPT.TOT & $447 \cdot 5$ & $367 \cdot 0$ & $361 \cdot 2$ & $424 \cdot 2$ \\
\hline & LWKR & $*$ & $408 \cdot 5$ & $401 \cdot 1$ & $468 \cdot 6$ \\
\hline & FCFS & $*$ & $449 \cdot 5$ & $409 \cdot 6$ & $*$ \\
\hline \multirow[t]{5}{*}{$7 \%$} & SPT & $573 \cdot 2$ & $359 \cdot 4$ & $394 \cdot 6$ & $480 \cdot 9$ \\
\hline & SPT/TOT & $*$ & $431 \cdot 7$ & $344 \cdot 7$ & $*$ \\
\hline & SPT.TOT & $535 \cdot 9$ & $422 \cdot 2$ & $396 \cdot 1$ & $587 \cdot 6$ \\
\hline & LWKR & $*$ & $430 \cdot 5$ & $424 \cdot 6$ & $661 \cdot 2$ \\
\hline & FCFS & $*$ & $498 \cdot 7$ & $450 \cdot 9$ & $*$ \\
\hline \multirow[t]{5}{*}{$10 \%$} & SPT & $*$ & $369 \cdot 3$ & $422 \cdot 5$ & * \\
\hline & SPT/TOT & $*$ & $476 \cdot 1$ & $551 \cdot 7$ & $*$ \\
\hline & SPT.TOT & $*$ & $475 \cdot 9$ & $467 \cdot 3$ & $*$ \\
\hline & LWKR & $*$ & $483 \cdot 6$ & $478 \cdot 2$ & $*$ \\
\hline & FCFS & $*$ & $530 \cdot 1$ & $517 \cdot 3$ & $*$ \\
\hline
\end{tabular}

* The system was saturated.

Table 10. Performance of the rules at varying AGV breakdown rates.

before, the relative priorities of parts waiting on the carousel were lower than the parts in the system. Thus, upon completion of any material transfer, an AGV first serviced the parts in system and then the input/output carousel as long as the destination station of a part were available. Later, this priority assignment was changed and the priorities of parts were made equal regardless of their physical locations in the system.

When the same experiment was repeated, it was noticed that the system seldom reached a stable behaviour at the high utilization levels (i.e. above $85 \%$ ). This was due to the fact that at high utilization rates (or high load levels), the system was periodically overloaded by the AGV system under the new priority scheme. In order to prevent this situation a kind of 'gate' was put into effect to limit the number of jobs released into the system. Table 11 illustrates the behaviour of the mean flowtime and its elements as the gate size (or number of jobs allowed in the system) is varied. 


\begin{tabular}{lrrrrrrr}
\hline & \multicolumn{7}{c}{ Gate size } \\
\cline { 2 - 8 } Elements of flowtime & \multicolumn{1}{c}{20} & 25 & \multicolumn{1}{c}{30} & 35 & 40 & 45 & 50 \\
\hline Input queue & $156 \cdot 8$ & $189 \cdot 4$ & $203 \cdot 8$ & $230 \cdot 1$ & $240 \cdot 0$ & $265 \cdot 2$ & $*$ \\
Output queue & $25 \cdot 3$ & $37 \cdot 1$ & $45 \cdot 2$ & $53 \cdot 8$ & $58 \cdot 4$ & $64 \cdot 8$ & $*$ \\
Machines & $100 \cdot 3$ & $100 \cdot 3$ & $100 \cdot 3$ & $100 \cdot 3$ & $100 \cdot 3$ & $100 \cdot 3$ & $*$ \\
Carousel & $431 \cdot 8$ & $141 \cdot 6$ & $76 \cdot 6$ & $61 \cdot 0$ & $59 \cdot 1$ & $62 \cdot 1$ & $*$ \\
Buffer & $0 \cdot 4$ & $3 \cdot 8$ & $7 \cdot 9$ & $17 \cdot 2$ & $31 \cdot 0$ & $53 \cdot 7$ & $*$ \\
AGV & $8 \cdot 7$ & $8 \cdot 9$ & $9 \cdot 1$ & $9 \cdot 4$ & $9 \cdot 7$ & $10 \cdot 2$ & $*$ \\
Blocking & $0 \cdot 2$ & $1 \cdot 5$ & $3 \cdot 7$ & $5 \cdot 5$ & $6 \cdot 1$ & $7 \cdot 9$ & $*$ \\
Flowtime & $724 \cdot 5$ & $482 \cdot 6$ & $446 \cdot 6$ & $477 \cdot 3$ & $504 \cdot 6$ & $564 \cdot 2$ & $*$ \\
\hline
\end{tabular}

$\dagger$ FCFS was used for scheduling of both machines and AGVs.

* The system was saturated.

Table 11. Elements of the mean flowtime as a function of gate size when the queue capacity is five $\dagger$.

\begin{tabular}{|c|c|c|c|c|c|c|c|}
\hline \multirow[b]{2}{*}{ Elements of flowtime } & \multicolumn{7}{|c|}{ Gate size } \\
\hline & 20 & 25 & 30 & 35 & 40 & 45 & 50 \\
\hline Input queue & $10 \cdot 6$ & $12 \cdot 8$ & $13 \cdot 8$ & $15 \cdot 5$ & $16 \cdot 2$ & $17 \cdot 8$ & $*$ \\
\hline Output queue & $1 \cdot 7$ & $2 \cdot 5$ & $3 \cdot 1$ & $3 \cdot 7$ & $3 \cdot 9$ & $4 \cdot 4$ & $*$ \\
\hline Buffer & $10 \cdot 6$ & $12 \cdot 8$ & $13 \cdot 8$ & $15 \cdot 5$ & $16 \cdot 2$ & $17 \cdot 8$ & $*$ \\
\hline Carousel & $28 \cdot 3$ & $5 \cdot 0$ & $5 \cdot 0$ & $4 \cdot 0$ & $3 \cdot 8$ & $4 \cdot 1$ & $*$ \\
\hline Input queue & $19 \cdot 8$ & $23 \cdot 2$ & $25 \cdot 1$ & $28 \cdot 1$ & $30 \cdot 1$ & $33 \cdot 8$ & $*$ \\
\hline In system & $48 \cdot 1$ & $32 \cdot 4$ & $30 \cdot 1$ & $32 \cdot 1$ & $33 \cdot 9$ & $37 \cdot 9$ & $*$ \\
\hline Mach. util. & $84 \cdot 5$ & $84 \cdot 5$ & $84 \cdot 5$ & $84 \cdot 5$ & $84 \cdot 5$ & $84 \cdot 5$ & * \\
\hline AGV util. & $69 \cdot 5$ & $72 \cdot 5$ & $73 \cdot 5$ & $76 \cdot 5$ & $78 \cdot 5$ & $82 \cdot 0$ & $*$ \\
\hline No. of blocking & 70 & 279 & 423 & 675 & 889 & 1112 & $*$ \\
\hline
\end{tabular}

$\dagger$ FCFS was used for scheduling of both machines and AGVs.

* The system was saturated.

Table 12. Average queue lengths, average utilization, and total number of blocking statistics as a function of gate size when the queue capacity is five $\dagger$.

The statistics for some of the system variables are also presented in Table 12. These results indicate that at lower gate values, where the system is underloaded, parts are spending a considerable amount of time in the input/output carousel, instead of being processed in the system. On the other hand, at higher gate values when the system is overloaded, the number of blockings increases or a locking situation occurs and AGVs that could unlock the system are tied up. In both cases, the flowtime drastically increases and its general behaviour as a function of the gate size becomes a convex curve. Thus, this gate performs a smoothing role by controlling the material flow into the system.

Under this new AGV priority scheme, the effect of different queue capacities on the flowtime was also investigated. As shown in Fig. 6, the lower flowtime performances was achieved at higher queue capacities. This is due to the fact that the number of blockings increases at low queue capacities. Consequently, a part cannot proceed to the next machine, which in turn increases the waiting time of 


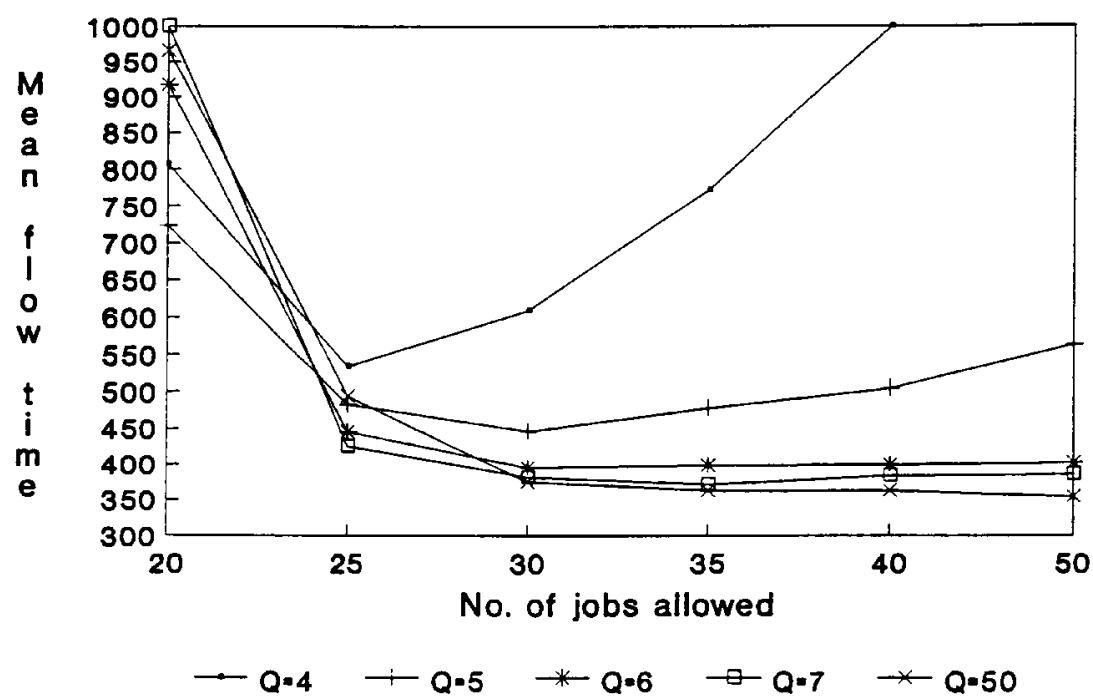

Figure 6. Mean flowtime vs no. of jobs allowed at different queue capacities.

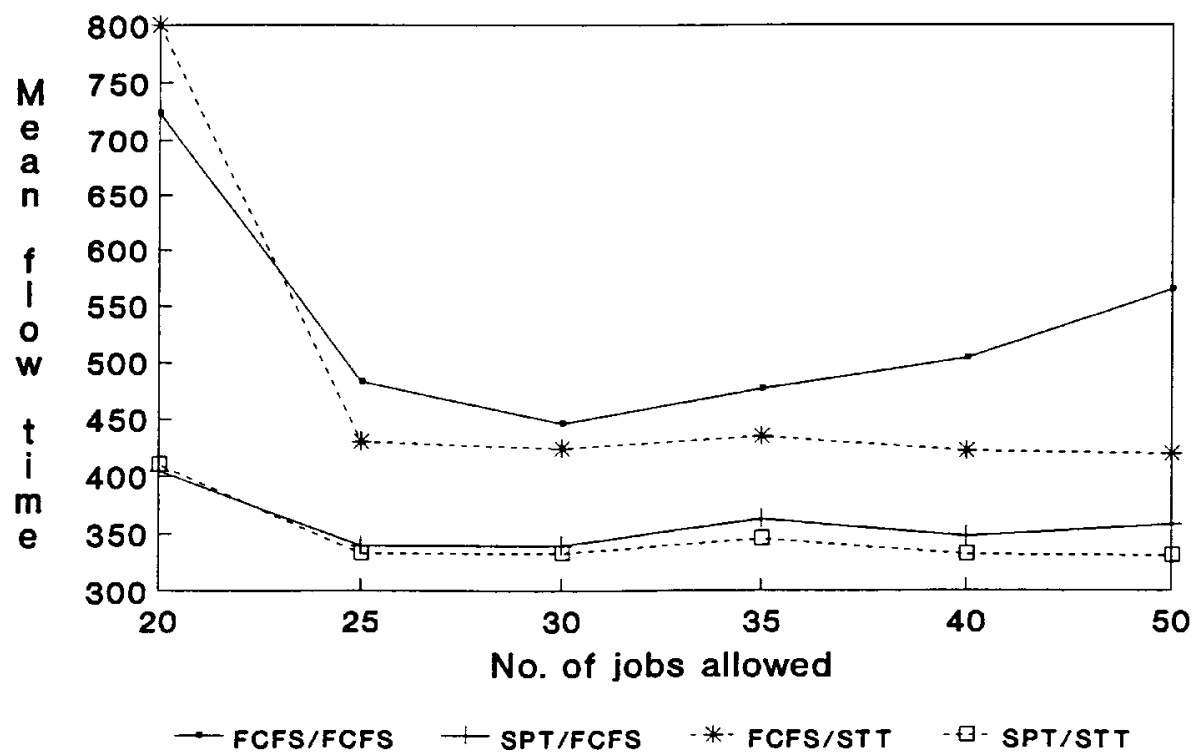

Figure 7. Mean flowtime performance of different machine and AGV scheduling rules.

out-going parts at workcentres and the input-output carousel. The optimal gate size is a function of the queue capacity of the workcentre.

The same experiments were also repeated with different machine and AGV rule combinations (Fig. 7). The results indicated that the mean flowtime was highly affected by the different combinations of machine and AGV scheduling rules. While SPT/STD performed best, FCFS/FCFS yielded the poorest performance of those tested. From the previous results, it is generally known that SPT is a better machine scheduling rule than the simple FCFS rule against the mean flowtime criterion. Thus, the combination of SPT and STD produced the best results. 


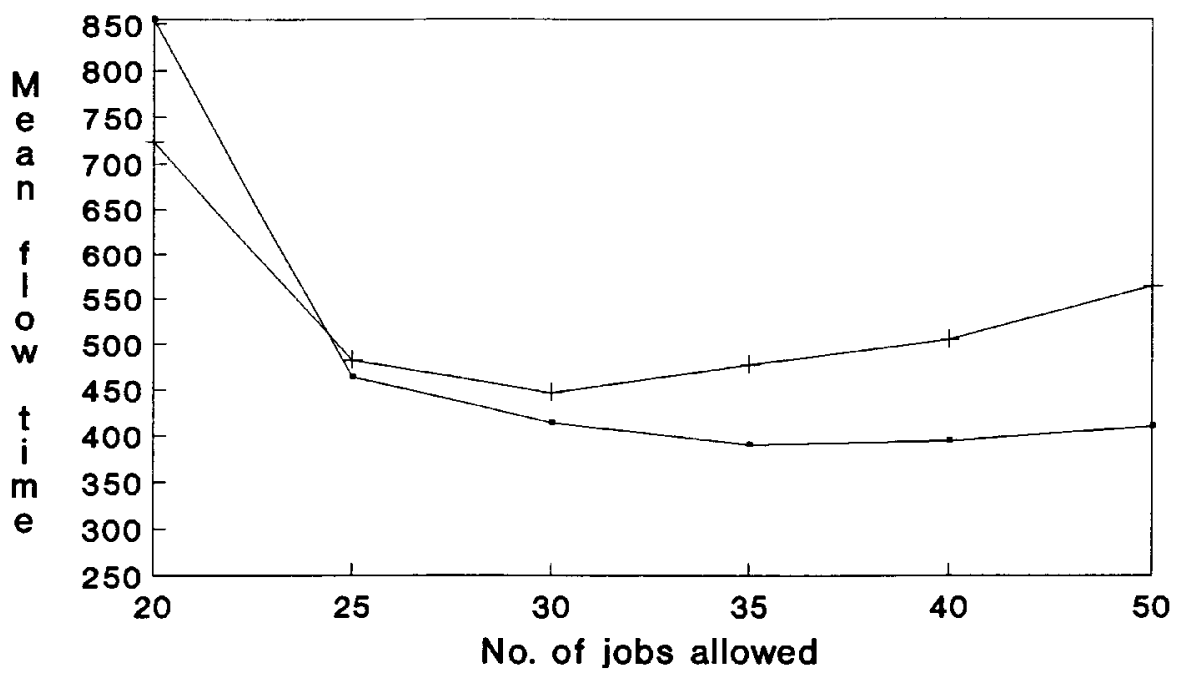

- ORIGINAL $\rightarrow$ AFTER CHANGE

Figure 8. Mean flowtime vs no. of jobs two priority scheme at queue size five.

Since some interesting results were obtained by changing the AGV priority, the original priority scheme was also tested at varying gate sizes. As illustrated in Fig. 8, when the original AGV priority scheme was used, the effect of the gate size on the flowtime was reduced and the system became more stable (i.e. the mean flowtime is insensitive to the number of jobs allowed). Therefore, this former priority assignment was planned to be used for the rest of the simulation results reported in this research.

In conclusion, knowing that an FMS is a tightly integrated system and has to operate at high utilization rates, early or late release of an order may adversely affect the system performance. Thus, it may be necessary to limit the number of jobs in the system in order not to cause excessive congestion and traffic on the shop floor. This result is also consistent with the work of Buzacott and Shantimumar (1993, chapter 8) that a certain amount of improvement in the performance of FMSs can be achieved by effective part loading and release (i.e. not releasing jobs immediately).

\section{Concluding remarks and suggestions for further research}

This study showed that the performance of FMSs can be improved considerably by using appropriate scheduling rules. This was even observed under very lightly loaded system conditions in which differences between the rules are found to be statistically significant. The results also indicated that scheduling of material handling systems (i.e. an AGV system) is as important as the machining subsystem. Other findings are as follows:

(1) The machine and AGV load levels appeared to be the major factors in determining the relative performances of the scheduling rules. As in the previous work (Sabuncuoglu and Hommertzheim 1992a, b), differences in the relative performances of the rules become more significant when the system load increases. However, deteriorations in the performance of the rules are more pronounced in the exponential case. This may be due to the higher 
variance of exponential distribution. The results of statistical tests also indicated that differences between the rules are significant over a large range of load or utilization levels (i.e. from $55 \%$ to $75 \%$ and above). In contrast to the previous works in the LQS was the most preferred rule, STD displayed the best performance in the exponential case. Among machine scheduling rules tested, SPT was still the best rule.

(2) Similarly to the previous work (Sabuncuoglu and Hommertzheim 1992a, b), the system experiences higher flowtimes as the queue capacities are reduced. At low queue capacities, AGV scheduling rules played a major role in the material flow due to a lack of decision possibilities for machine scheduling rules in short queue length situations. We also note that LQS improves the system flow times more than STD at short queue capacities.

(3) The performance of the rules deteriorated as the down time percentage increases. The results also indicated that the adverse effect of machine failure on the system performance is more than the AGV failure. Moreover, it was noted that consideration of breakdowns change the relative performances of the AGV rules. Specifically, STD produced better mean flowtime performances than LQS when the down time percentage was low, but the results were reversed when the down time percentage increased. This is due to the fact that LQS responds faster than STD to blocking events as the downtime percentage increases.

(4) We noted that both variance reduction and randomized block design are very effective in separating the performances of scheduling rules at even lightly loaded system conditions.

(5) The mean flowtime performance of the rules is affected by the variances of the operation time distribution. As compared to our previous studies, the system is saturated for most of the scheduling rules at the moderately loaded shop conditions (i.e. 80 or $83 \%$ utilizations) due to the high variance of the exponential distribution.

(6) The performance of the system was also very sensitive to the type of AGV priority scheme used. In an FMS, parts wait for an AGV at different places in the system such as workcentres, input/output carousel, etc. Prioritizing methods for the AGVs can significantly affect the system performance. For example, in the FMS simulated, the system flowtime was improved significantly by giving higher priority to the parts waiting in the system rather than the parts on the input/output carousel. This also shows the importance of order review-release for FMSs; an effective controlled release policy (i.e. releasing the jobs to the system in a controlled manner) can reduce congestion on the shop floor and improve the system performance.

The results presented in this paper are valid under the experimental conditions described earlier. Hence, there is a need for further research to develop new rules and continue testing the existing ones under different FMS configurations and experimental conditions. Such research should address the impact of varying system parameters (i.e. changes in arrival rates, variation in processing times), several types of flexibility measures, different FMS configurations and AGV networks. It may also be interesting to compare the performance of the rules with the off-line scheduling algorithms under dynamic and stochastic shop conditions. Another research direc- 
tion would be to test scheduling rules (both machine and AGV rules) with several order review/release mechanisms and understand their relationships.

\section{References}

Acree, E. S., and Smith, M. L., 1985, Simulation of a flexible manufacturing system-applications of computer operating system techniques. The 18th Annual Simulation Symposium 1985 (Tampa, FL: IEEE Computer Society Press), pp. 205-216.

Basnet, C., and Mize, J. H., 1994, Scheduling and control of manufacturing systems: a critical review. International Journal of Computer Integrated Manufacturing, 7, 340-355.

Blackstone, J. H., Phillips, D. T., and Hogg, G. L., 1982, A state-of-the-art survey of dispatching rules for manufacturing job shop operations. International Journal of Production Research, 20 (1), 27-45.

Buzacott, J. A., and Shantikumar, J. G., 1993, Stochastic Models of Manufacturing Systems (Englewood Cliffs, NJ: Prentice Hall).

Сноi, R. H., and Malstrom, E. M., 1988, Evaluation of traditional work scheduling rules in a flexible manufacturing system with a physical simulator. Journal of Manufacturing Systems, 7 (1), 33-45.

Сно, H. C., Jaw, T. J., and Chen, S. K., 1988, Sequencing in flexible manufacturing systems and other short queue-length systems. Journal of Manufacturing Systems, 7(1), 1-7.

Conway, R. W., Maxwell, W. L., and Miller, L. W., 1967, Theory of Scheduling (Reading, MA: Addison-Wesley).

Denzler, D. R., and Boe, W. J., 1987, Experimental investigation of flexible manufacturing system scheduling rules. International Journal of Production Research, 25(7), 979-994.

Egbelu, P. J., and Tanchoco, J. M. A., 1984, Characterization of automated guided vehicle dispatching rules. International Journal of Production Research, 22 (3), 359-374.

Ishit, N., and Talavage, J. J., 1991, A transient based real-time scheduling algorithm in FMS. International Journal of Production Research, 29 (12), 2501-2520.

Kir an, A. S., and Smith, M. L., 1984a, Simulation studies in job shop scheduling - a survey. Computers and Industrial Engineering, 8(2), 87-93.

Kiran, A. S., and Smith, M. L., 1984b, Simulation studies in job shop scheduling - performance of priority rules. Computers and Industrial Engineering, 8(2), 95-105.

Kusia k, A., and Chen, M., 1988, Expert systems for planning and scheduling manufacturing systems. European Journal of Operations Research, 34(3), 113-130.

Mackay, K. N., Safayeni, F. R., and Buz acott, J. A., 1988, Job-shop Scheduling Theory: What is relevant? Interface, $\mathbf{1 8}(4), 84-90$.

Montazeri, M., and van Wassenhove, L. N., 1990, Analysis of scheduling rules for an FMS. International Journal of Production Research, 28(4), 785-802.

Panwalkar, S. S., and Iskander, W., 1977, A survey of scheduling rules. Operations Research, $25(1), 45-61$.

Pegden, C. D., Shannon, R. E., and Sadowski, R. P., 1990, Introduction to Simulation using SIMAN (New York: McGraw-Hill).

Rachamadugu, R., and Stecke, K. E., 1994, Classification and review of FMS scheduling procedures. Production Planning and Control, 5, 2-20.

Rachamadugu, R., Nandkeolyar, U., and Schriber, T. J., 1993, Scheduling with sequence flexibility. Decision Science, 24, 315-341.

Raman, N., Talbot, F. B., and Rachamadugu, R., 1989, Due date based scheduling in a general flexible manufacturing system. Journal of Operations Management, 8, 115-132.

Ravi, T., Lashrari, R. S., and DutTa, S. P., 1991, Selection of scheduling rules in FMSs-a simulation approach. International Journal of Advanced Manufacturing Technology, $\mathbf{6}$, 246-262.

Sabuncuoglu, I., and Hommertzheim, D. L., 1992a, Experimental investigation of FMS machine and AGV scheduling rules against the mean flowtime criterion. International Journal of Production Research, 30, 1617-1635.

Sabuncuoglu, I., and Hommertz heim, D. L., 1992b, Dynamic dispatching algorithm for scheduling machine and AGVs in a flexible manufacturing system. International Journal of Production Research, 30, 1059-1080. 
Sabuncuoglu, I., and Hommertzheim, D. L., 1993, Experimental investigation of FMS due-date scheduling problem: evaluation of machine and AGV scheduling rules. International Journal of Flexible Manufacturing Systems, 5, 301-324.

Sabuncuoglu, I., and Hommertzheim, D. L., 1995, Experimental investigation of an FMS due-date scheduling problem: an evaluation of due-date assignment rules. International Journal of Computer Integrated Manufacturing, 8, 133-144.

Sabuncuoglu, I., and Karabur, S. A., 1997, A beam search-based algorithm and evaluation of scheduling approaches. IIE Transactions (forthcoming).

Stecke, K. E., 1983, Formulation and solution of nonlinear integer production planning problems for flexible manufacturing systems. Management Science, 29, 273-287.

Stecke, K. E., and Solberg, J., 1981, Loading and control policies for flexible manufacturing system. International Journal of Production Research, 19, 481-490.

TAng, L. L., Y IH, Y., and Liu, C.-Y., 1993, A study on decision rules of a scheduling model in an FMS. Computers in Industry, 22, 1-13.

Ulusoy, G., and Bilge, U., 1993, Simultaneous scheduling of machines and automated guided vehicles. International Journal of Production Research, 31, 257-2873.

Wu, S. D., and Wysk, R. A., 1988, Multi-pass expert control system - a control/scheduling structure of flexible manufacturing cells. Journal of Manufacturing Systems, 7 (2), 107120.

Wilk Inson, L., 1987, S YSTAT Reference Manual, SYSTAT. 